\title{
Le vaudeville sous la Deuxième République : une arène ouverte aux passions politiques ?
}

Janice Best, Université Acadia

L'un des premiers actes du Gouvernement provisoire issu de la Révolution de février 1848 fut de supprimer la Commission d'examen des ouvrages dramatiques. Désormais, les auteurs avaient la liberté de faire représenter ce qui leur semblait bon sur les planches. Le résultat immédiat de cette liberté fut un foisonnement de pièces à sujets politiques, notamment au théâtre du Vaudeville, qui tournaient en ridicule les chefs du gouvernement, les députés et les républicains, devant des salles enthousiastes (Hemming, Malandain, Yon). Dès le 30 juillet 1850, cependant, une nouvelle loi rétablissait l'examen préalable des ouvrages dramatiques. Dans cet article, trois vaudevilles joués pendant cette période de liberté d'expression seront examinés, notamment La propriété, c'est le vol! de Jules Clairville et Jules Cordier (jouée au théâtre du Vaudeville en novembre 1848), La foire aux idées par Leuven et Brunswick (représenté au théâtre du Vaudeville en janvier 1849), et Les représentants en vacances de Clairville et Cordier (représenté au théâtre du Gymnase-Dramatique en septembre 1849) afin de souligner le rôle de contestation politique que le vaudeville joua pendant cette courte période de liberté.

Les commentaires politiques ne disparurent pas après le retour de la censure, mais furent l'objet d'une attention accrue de la part des autorités, désireux de rendre le théâtre « un lieu de repos et de distraction et non pas une arène ouverte aux passions politiques » (Archives nationales, F/21/4635). J'examinerai donc, dans un deuxième temps, trois vaudevilles joués après le retour de la censure, en particulier Les pavés sur le pavé de Leuven, Lhérie et Beauplan, (représenté au théâtre du Vaudeville en septembre $1850^{1}$ ), Une clarinette qui passe d'Eugène Labiche et Marc-Michel (joué au théâtre des Variétés en janvier 1851) et Les effrayés de Théodore Muret et Gaston de Montheau (pièce d'abord ajournée et enfin jouée au théâtre des Variétés en novembre 1851), afin de cerner à la fois ce processus de répression et la façon dont les auteurs contournaient ces contraintes à leur liberté d'expression.

Connu pour sa comédie basée sur des surprises et des quiproquos ainsi que pour l'utilisation des «timbres » (ou couplets chantés sur des airs empruntés), le vaudeville vivait de nouveautés et sollicitait une participation active de la part des spectateurs (Martin 69, Terni 222). Pour cette raison, les censeurs prêtèrent une attention particulière à ce type de pièce, assistant 
même aux représentations afin de veiller à ce que les changements qu'ils avaient demandés soient effectivement réalisés. Comme l'écrit Olivier Bara : «le spectacle vaudevillesque déploie ses effets et produit des significations à partir de sa musique, de ses chansons comme de ses pantomimes ou de ses danses. Son langage scénique foisonnant, mobile, en constant excès spectaculaire, effraie les censeurs...»(159). Cette proximité avec l'actualité fit que les directeurs de théâtre étaient sans cesse à la recherche de nouvelles pièces pour renouveler leur répertoire. Les auteurs de vaudevilles étaient donc habitués à écrire vite et à s'adapter à des circonstances changeantes. De tous les genres théâtraux, le vaudeville était donc un des plus aptes à évoluer en fonction d'une situation politique fluide.

Malgré la réouverture des théâtres dès la mi-mars 1848, le public se fit rare pendant les premiers mois de la nouvelle République, en partie à cause de l'incertitude politique et en partie en raison de la crise financière qui fut une des principales causes de la révolution, et à laquelle on cherchait toujours des solutions. Plusieurs théâtres se virent menacer de faillite. Afin de faire revenir le public, les vaudevillistes ne tardèrent pas à exploiter le goût du public pour les nouveautés et les actualités du jour en incorporant des allusions aux barricades de février dans leurs pièces.

\section{La liberté d'expression}

À l'Opéra national (l'ancien Cirque olympique), on vit dès la mi-mars Les barricades de 1848, un opéra-comique en deux actes de Brisebarre et Déaddé. Au théâtre du Luxembourg on donna Les barricades des 23 et 24 février, un vaudeville en un acte, et le 11 mars 1848 au Gymnase on joua Les filles de la Liberté, à-propos vaudeville de Clairville et Cordier mettant en scène des femmes en robes légères, coiffées de bonnets phrygiens, incarnant la Liberté de la presse, la Liberté des théâtres, la Liberté des votes, la Liberté de la parole, la Liberté des champs, la Liberté des ponts, et «deux ou trois autres petites Libertés, sans oublier la Liberté de l'émeute » (Le constitutionnel 20 mars 1848). Au théâtre de la République, la tragédienne Rachel, alors au sommet de sa gloire, chantait La marseillaise après chaque représentation.

À ces pièces consacrées aux événements révolutionnaires, succéda toute une série d'ouvrages exploitant l'engouement pour les banquets, les clubs politiques et les nouvelles idées démocratiques. Les auteurs les plus à succès pendant cette période furent sans conteste Cordier et Clairville. Suite à leur triomphe avec Les filles de la Liberté, ils abordèrent d'autres sujets 
politiques. Dans Le club des maris et le club des femmes, vaudeville en un acte joué au théâtre du Vaudeville le 4 juin 1848, par exemple, on voit plusieurs femmes, fâchées parce que leurs maris les ont désertées, qui cherchent à fonder un club de femmes mariées. À l'encontre de leurs maris, qui s'occupent d'améliorer leur sort, les femmes veulent s'occuper «de choses raisonnables, utiles... » (sc. 2). Lorsqu'elles apprennent que leurs maris vont débattre la question « La femme est-elle nécessaire à l'homme », elles décident de se venger. Elles revendiquent leur liberté et leur indépendance, et veulent tout révolutionner en renversant l'autorité des hommes. Désormais, l'époux devra l'obéissance à la femme et devra faire et la cuisine et le ménage (sc. 3). Tous se réconcilient à la fin, cependant. Les maris sont d'accord pour fermer leur club, les femmes le leur, et le bonheur conjugal est restauré.

Quelques mois plus tard, également sur le théâtre du Vaudeville, Clairville et Cordier firent représenter une folie socialiste en trois actes et sept tableaux qui tournait en ridicule les idées de Pierre-Joseph Proudhon intitulée La propriété, c'est le vol! Cette pièce commence par un prologue où un solliciteur explique au public que le but de la pièce est de faire rire « le public de la veille / Et le public du lendemain », une référence à l'expression «républicains de la veille » et « républicains du lendemain » pour désigner ceux qui avaient de vraies convictions démocratiques avant la révolution et ceux qui les avaient adoptées après la révolution par opportunisme.

Ce prologue fait place à un tableau montrant Adam et Ève au paradis terrestre, tentés par un serpent à tête humaine portant des lunettes ${ }^{2}$, fâché d'avoir été supplanté par un homme et une femme qui prétendent que tout ce qui était autrefois à lui appartient maintenant à eux. Adam est donc présenté comme le premier propriétaire et le serpent jure de se venger de lui. La suite de l'action se passe entre 1848 et 1854. La pièce prend donc son point de départ dans l'actualité politique du jour mais se projette vers un avenir imaginaire. Chaque acte fait se confronter deux personnages principaux, le serpent à lunettes et Bonnichon, un riche propriétaire qui ressemble étrangement à Adam. Le serpent poursuit sa vengeance jusqu'à la fin de la pièce, s'attaquant sans répit au principe de la propriété, défendu par Bonnichon, et annonçant la fin de la bourgeoisie et la fin du monde.

En septembre 1849, Clairville et Cordier firent représenter une autre comédie-vaudeville à sujet politique, cette fois-ci au théâtre du Gymnase-Dramatique, Les représentants en vacances. L'action de cette pièce a lieu lors d'une prorogation de la Chambre des députés. À 
cause des vacances, sept cent cinquante maris sont lâchés par la France. Trois amis de collège, le comte de Blossac, un monarchiste, Dumoulin, un représentant de gauche, et un modéré, Morisset, se réconcilient dans la malle poste qui les emmène à la campagne. Ils jurent de ne plus parler de politique pendant leurs vacances au risque de payer vingt-cinq francs d'amende. Mais trois délégués arrivent, envoyés par les électeurs du département, le premier réclamant le droit au travail, l'impôt progressif et le partage des biens; le deuxième, l'ordre avant tout; et le troisième, la restauration de la monarchie. Devant le mécontentement de leurs électeurs, les représentants n'ont d'autre choix que de retourner à Paris afin de retrouver le calme.

Les nouvelles idées et institutions politiques se reflètent ainsi dans ces trois pièces, mais se trouvent mélangées à d'autres éléments plus traditionnels au vaudeville. Dans Le club des maris et le club des femmes, par exemple, l'enthousiasme pour les clubs et les débats politiques crée le point de départ et le conflit principal, mais il sert également à faire avancer d'autres éléments de l'intrigue. Lors de la première réunion du club des femmes, par exemple, plusieurs avouent qu'un jeune homme leur fait la cour. Lorsqu'elles découvrent qu'il s'agit du même homme, un certain Saint-Estève ${ }^{3}$, elles jurent de se venger. Pendant qu'au club des maris on procède à l'élection d'un nouveau président qui, selon leurs statuts, doit être « le plus compromis par sa femme, le plus menacé dans son bonheur » (sc. 8), les femmes préparent leur vengeance.

Entraîné par elles dans un piège, Saint-Estève se fait tabasser. Il se venge en livrant les lettres d'amour écrites par lui à tous les membres du club des maris. Les maris, à leur tour, jurent la revanche. Mais la femme de Saint-Estève finit par convaincre les autres maris qu'ils sont tous coupables d'avoir délaissé leurs femmes. Au lieu de chercher à se venger, ils devraient plutôt fermer leur club et écrire des billets doux à leurs épouses. On voit donc associés à des sujets d'actualité politique dans cette pièce, d'autres thèmes plus typiques du vaudeville, tels la vengeance, la jalousie et l'infidélité conjugale.

Dans La propriété, c'est le vol !, chaque acte met en scène une nouvelle étape de la révolution socialiste. Au deuxième acte, qui se passe dans un salon en 1848, Bonnichon et plusieurs de ses amis boivent à la santé de la réforme électorale, « une révolution socialiste par la garde nationale dans l'intérêt des propriétaires » (II, 1). Un serpent à lunettes interrompt les festivités pour annoncer que « ce n'est pas seulement la réforme électorale, c'est la République qui vient d'être proclamée. [... ] La bourgeoisie est détrônée... le peuple est roi !» (II. 2). Les autres tableaux nous montrent Bonnichon en 1852, l'année où le droit au travail vient d'être 
décrété par le nouveau président de la République, ensuite en 1853, où un autre décret du même président maintenant déchu abolit l'aristocratie et établit l'égalité de tous.

L'acte final montre la fin du monde en 1854. Le théâtre est couvert d'herbages et de ruines et au fond on voit la Bourse, également en ruines. Sur un poteau se trouve une affiche annonçant l'ouverture de la chasse. Bonnichon paraît, couvert d'une peau d'animal. Il ne reste que lui et le serpent au monde et le serpent ne tarde pas à donner la mort au dernier des propriétaires. Malgré cette fin apocalyptique, le dénouement est heureux. Le monde renaît, ainsi que la propriété et les propriétaires. Le serpent n'était pas méchant, seulement myope et une nouvelle paire de lunettes lui permet de voir l'utilité de la propriété.

Le point de départ des Représentants en vacances, le retour à la campagne des trois députés parisiens, renverse un des thèmes traditionnels du vaudeville, celui des provinciaux débarqués à Paris et dont on se moque. Dans cette pièce, ce sont les Parisiens et leurs nouvelles idées qui sont les objets de ridicule, car malheureusement pour les trois amis qui cherchaient surtout à s'échapper aux débats politiques, les idées de la capitale les ont précédés et ont déjà transformé la campagne. Pendant que leurs représentants travaillaient à Paris, les citoyens du département ont lu leurs discours dans le journal et s'en sont inspirés. Les légitimistes sont devenus plus légitimistes que la légitimité, les républicains plus républicains que la République et les modérés sont devenus des modérés «excessifs» (sc. 4). Tandis que les légitimistes conspirent et les républicains préparent une émeute, les modérés tentent de maintenir l'ordre. Devant le peu d'enthousiasme de leurs représentants, tous les accusent d'avoir manqué à leurs principes et d'être trop réactionnaires.

De retour chez eux, les députés doivent défendre leurs maisons, jardins et épouses contre les nouvelles tendances démocratiques qui remettent en question le bien fondé de la propriété et du mariage. Ils oublient vite leurs principes politiques face à la destruction de leurs biens et de leur famille. Si les élus dans Les représentants en vacances décident de repartir à la capitale, c'est parce qu'ils ont été choqués par les effets dévastateurs que leurs discours politiques ont produits sur la population locale. C'est avec un espoir d'entente et de réconciliation qu'ils quittent la campagne.

Dans Le siècle du 24 septembre 1849, le critique dramatique Matharel loue l'esprit de conciliation de la pièce :

[...] c'est la première œuvre depuis février ayant un caractère politique qui ne soit 
pas agressive pour la révolution. Les réactionnaires, les rouges, les légitimistes parlent et entendent un langage convenable, plein de goût, de mesure, et de bon sens. C'est un très gros fait que la représentation de cette pièce ; elle a toute l'importance d'un acte politique, et le succès de cette comédie est de nature à donner à réfléchir à plus d'un esprit sérieux.

Si les idées nouvelles créent le désordre dans les provinces, mettant en péril les valeurs bourgeoises de propriété et de travail, elles servent aussi à faire avancer une intrigue amoureuse plus traditionnelle dans le vaudeville car on y retrouve un couple de jeunes amoureux, Armand et Cécile, dont la politique fait obstacle au bonheur. Lorsque Dumoulin et Blossac découvrent Cécile et Armand ensemble, par exemple, ils leur interdisent de se fréquenter pour ne pas trahir leurs opinions politiques.

Cécile et Armand tentent d'abord de profiter des convictions politiques de leurs parents pour leur faire lever l'interdiction. Cécile - en réalité une légitimiste - fait semblant de lire $L e$ droit au travail et fait croire à Dumoulin que les élèves de son pensionnat sont secrètement abonnées à La réforme et au National, deux journaux de gauche. Et Armand - qui est réellement de gauche - fait croire à De Blossac qu'il est légitimiste. Charmés, les deux parents sont sur le point de donner leur aval à l'union de leurs enfants lorsqu'ils s'aperçoivent de l'imposture. Une nouvelle querelle éclate entre le républicain et le légitimiste : «DUMOULIN. Ton parti, c'est l'obscurité ! DE BLOSSAC. Le tien, c'est l'incendie !» (III, 14).

Ce n'est que lorsqu'un triple contingent d'électeurs mécontents arrive sous leurs fenêtres et font un triple charivari que les deux amis d'enfance comprennent qu'ils allaient encore se séparer pour des électeurs ingrats. Jurant de s'unir et de former une alliance pour le bonheur de la France, ils donnent enfin leur aval au mariage de leurs enfants. L'invasion des valeurs de la capitale dans l'espace de la province aboutit dans cette pièce par la création d'un nouvel espace de l'avenir, celui où les valeurs principales sont le bon sens, l'amour de la patrie et la réconciliation.

Le succès de ces trois pièces nous en dit long sur le public théâtral de cette époque, que ces allusions aux clubs politiques, aux théories de Proudhon et aux représentants nouvellement élus faisaient rire. Pouvoir parler des hommes politiques vivants et des débats du jour était une liberté nouvelle, interdite sous le régime de la censure préalable. Selon Jules Janin, La propriété, c'est le vol ! était un «adorable vaudeville», honnête, naïf, plaisant et amusant. (Le journal des débats, 4 décembre 1848). Le chroniqueur du Constitutionnel constata lui aussi le succès de la 
pièce :

Faisons d'abord nos très sincères compliments au théâtre du Vaudeville; il était mort, et le voici ressuscité ; le silence régnait dans sa salle déserte, et l'oubli ensevelissait ses œuvres une à une ; maintenant, il se réconcilie de jour en jour avec la foule, repeuple son parterre et ses loges, et commence à faire un peu de bruit [...] Cette amusante épigramme, armée de grosses pointes et de sept tableaux, continuera donc la prospérité renaissante du Vaudeville, et, si je suis bien informé, la foule est aux portes depuis huit jours. Il y a assez de gaieté et de verve dans la pièce, et suffisamment de scandale par allusion, pour justifier cette curiosité et l'entretenir longtemps. (1848.12.04).

Le goût du public pour ces sujets d'actualité ne fit que s'accroître tout au long de la courte durée de la Deuxième République. L'année 1849 en particulier fut marquée par une succession de pièces satirisant les nouvelles idées politiques. Une série de sketches intitulés $L a$ foire aux idées de Leuven et Brunswick, représentés au théâtre du Vaudeville en janvier 1849, créèrent la polémique. Le Caprice et le Capital jouent les rôles principaux dans cette pièce. Le Caprice veut louer un logement. Le loyer était auparavant de dix mille francs, mais vu la crise financière dans laquelle se trouve la France, le propriétaire consent à le louer pour dix-sept francs cinquante centimes. Survient le Capital, effrayé par des pillards qui ont essayé de lui voler ses écus. Ses créanciers ne veulent lui payer l'argent qu'ils doivent qu'au dixième président prochain, ce qui lui crée un dilemme.

Afin de sauver le Capital de la ruine, l'Idée, mère du Caprice, propose de fonder un journal, dont le Capital sera l'actionnaire, et qui publiera toutes les nouvelles de Paris à l'instant où elle se produisent. Les premières nouvelles ainsi publiées sont avant tout contradictoires. Un moment la Bourse est à la hausse et la confiance renaît, la seconde d'après la Bourse est à la baisse et la confiance s'en va. Survient alors Mademoiselle France, qui a été bien malade, mais qui est maintenant convalescente.

Comme on peut le constater, la pièce, qui s'apparente à la tradition des revues de fin d'année où l'on satirise les événements et personnalités marquants de l'année, contenait de nombreuses allusions à l'actualité politique du jour, aux barricades, au libre-échange, aux clubs politiques, aux décrets du gouvernement, et même à la Constitution, qui limitait la présidence à un seul mandat. L'œuvre se termine, non pas par un dénouement heureux, mais par la promesse d'un nouveau journal chaque mois. Le public a beaucoup applaudi la pièce. Janin, dans sa chronique du Journal des débats du 22 janvier 1849 en fit la description : 
Cette Foire aux idées égale déjà le succès de la Propriété, c'est le vol ! La salle est pleine, la foule s'écrase aux portes. C'est une si charmante vengeance, en effet, quand on ne pense qu'au plaisir fugitif de l'heure présente, de voir traîner aux gémonies de la chanson les grands hommes et les grandes choses du lendemain! [...] si vous pouviez assister à la joie, au bonheur, aux extases du public qui prête l'oreille à ces vives satires !

D'autres critiques furent moins enthousiastes, jugeant la pièce réactionnaire et dangereuse. D'autres encore se scandalisèrent du costume d'Ève, considéré indécent. Les auteurs de La foire aux idées tinrent leur promesse et revinrent au théâtre avec encore trois numéros au cours de l'année 1849, le deuxième le 25 mars, le troisième le 23 juin et le quatrième le 13 décembre, devant des salles toutes aussi enthousiastes.

\section{Le retour de la censure}

Le décret qui abolit de fait la censure préalable des ouvrages dramatiques en 1848, ne mentionna pas spécifiquement le théâtre, mais abrogea la loi du 9 septembre $1835^{4}$. Ce faisant, le gouvernement avait promis une réforme au complet de l'organisation des théâtres. Une Commission du Conseil d'État fut créée, chargée de préparer une « loi sur les théâtres ». Cette Commission organisa une série d'auditions à l'automne 1849 , entre le 21 septembre et le $1^{\text {er }}$ octobre. Trente-deux personnalités du monde littéraire et dramatique furent entendues. La loi du 30 juillet 1850 rétablissait la censure par 352 voix pour et 192 contre, sans qu'il y ait eu un grand débat. L'article 1 de cette loi prévoyait une loi spéciale sur les théâtres qui devait être présentée dans le délai d'une année. Cette loi ne vit jamais le jour, et, en attendant, tout ouvrage dramatique devait obtenir «l'autorisation préalable du ministère de l'Intérieur à Paris, et du préfet dans les départements »(Le constitutionnel, 31 juillet 1850). De provisoire, la loi devint définitive, prorogée le 31 juillet 1851 et une deuxième fois le 31 décembre 1852.

Lorsqu'on rétablit la censure en 1850, la liste des directives fut claire : en général, les censeurs devaient combattre les tendances des auteurs à chercher des situations et des effets scéniques «dans l'antagonisme des classes inférieures et des hautes classes », « dans les attaques contre le principe d'autorité, contre la religion, la famille, la magistrature, l'armée, en un mot contre les institutions sur lesquelles repose la société » ou encore «dans la peinture plus ou moins hardie des mœurs dépravées des femmes galantes et de la vie de désordre » (AN, F/21/4635). Ils faisaient exception, en revanche, lorsque ces sujets étaient abordés dans des pièces comiques, citant «l'espèce de licence tolérée ordinairement dans ce genre d'ouvrage » 
(Rapport Claudine, Archives nationales F/21/990) et les «droits du vaudeville français », (Rapport Vente d'un riche mobilier, AN F/21/979 ; Best 254).

\section{Suppressions et atténuations}

Le goût du public pour les pièces à thème politique ne disparut pas avec le retour de la censure. Pendant plusieurs années encore les auteurs cherchèrent à insérer des allusions et les censeurs firent de leur mieux pour éliminer de telles références, tout en respectant le droit des vaudevillistes de continuer à satiriser l'actualité. Dans Les pavés sur le pavé, vaudeville en un acte, par exemple, les auteurs abordèrent la question des transformations de Paris entreprises dès 1850 par Louis-Napoléon Bonaparte afin de faire des allusions à la révolution de 1848 et à d'autres bouleversements politiques récents. Dans le vaudeville en un acte Les effrayés, l'action se passe dans un village à soixante kilomètres de Paris où un écrit incendiaire effraie les habitants par de sinistres prédictions pour l'année 1852. Le maire du village, Raimbert, tente de calmer les esprits en faisant jeter au feu les publications alarmistes. Cette pièce reprend ainsi le thème de l'incertitude politique à des fins comiques. Finalement, dans la comédie vaudeville en un acte Une clarinette qui passe, c'est le thème de l'inégalité entre les classes qui crée le conflit principal.

Le vaste projet de réaménagement urbain, commandité par l'empereur Napoléon III et dirigé par son préfet de la Seine, le baron Haussmann, établit le point de départ de l'action dramatique des Pavés sur le pavé. La phase initiale de ce projet visait l'agrandissement de la rue de Rivoli, mais comprenait plus tard, entre 1853 et 1870 , la démolition des vieux quartiers médiévaux insalubres de la capitale, la construction de larges avenues, de parcs et de places, l'annexion des banlieues autour de Paris, et la construction de nouveaux égouts, fontaines et aqueducs.

Les pavés sur le pavé met en scène Gros Caillou et Mastoc, tous deux des pavés de Paris, dont l'existence est sérieusement menacée. Ils sont traqués par un ennemi formidable, «un Anglais, né en Écosse, qui a pris un train de plaisir pour venir [les] ennuyer» (sc. 1). Cet Anglais, qui s'appelle Macadam, veut que les pavés le respectent car selon lui il est leur nouveau maître : «Mauvais ou bon, le vieux s'efface / Je suis nouveau, je vous remplace / C'est la mode aujourd'hui, ma foi !» (sc. 1). Gros Caillou conclut qu'il s'agit encore d'une révolution, à quoi devait à l'origine répondre Mastoc : «À quoi bon ?... on vient d'en faire une qui est toute 
fraîche ?», réplique supprimée par le gouvernement. La réponse de Gros Caillou, qui faisait également allusion aux récents événements politiques, fut elle aussi censurée : «Pardieu! pourquoi fait-on les révolutions ?... pour prendre la place des autres !» (sc. 1).

Si ces deux références à la révolution politique et sociale qui venait de tout bouleverser en France furent supprimées, il restait clair cependant qu'un nouvel ordre politique venait de s'installer dans les rues de Paris. C'est ce que souligna Janin dans son compte rendu de la pièce paru dans le Journal des débats (1850.09.09) :

Comment [...] on arrache le pavé de nos rues; comment! on ôte à l'émeute cette fiche de consolation; comment ! on prive la liberté de ses espérances les plus prochaines, le pavé ?, le pavé, qui est la base mobile des changements populaires ! Le pavé ! mais vous ne savez donc pas ce qui peut sortir d'un pavé ! La lumière en sort, et avec la lumière, l'affranchissement des nations !

Malgré les suppressions imposées par les censeurs, la pièce ne perdit pas toutes ses connotations politiques et continuait à souligner une transformation sociale importante. Dans les scènes finales, par exemple, on insiste sur les mauvaises conséquences de l'absence des pavés. Tout va de mal en pire. Les demoiselles de paveurs envisagent de quitter la France pour retrouver du travail. Et Gros Caillou se lamente d'être sans travail après trois cents ans de service durant lesquels lui aussi a vu bien des événements.

Au moment où Gros Caillou et les autres s'apprêtent à partir, Alfred, un commis, sort de chez lui, se plaignant du tourbillon de poussière qui l'empêche de se tenir dans son magasin. Macadam propose alors d'arroser le macadam. Lorsque Gros Caillou lui fait remarquer que cela ne ferait encore plus de poussière, un autre personnage s'exclame : «Écoutez, nous ne sortirons de là qu'avec des balais ». À la vue des balais, Gros Caillou comprend enfin la solution : «Il vient de me pousser une idée magnifique ! mirifique ! chorégraphique! je ne suis plus pavé ! je donne ma démission en masse... je me nomme premier balayeur de l'Europe ... en voilà de l'ouvrage !» (sc. 16). Tout le monde se met donc au travail, les pavés comme les paveurs, dont la mission est désormais de débarrasser le sol d'un «tas de choses désobligeantes », tels les «pièces immorales, feuilletons insalubres, petits livres malsains, entrefilets incendiaires » (sc. 16). La pièce se termine sur une note patriotique. Nettoyer la patrie est jugé par tous le sort le plus doux et le plus digne. Gros Caillou résume le sentiment général dans un vaudeville final, adressé au public: «Nous ne voulons en tous temps / attaquer que les méchants / à tous les mauvais français / ennemis de l'ordre et de la paix » (sc. 16). 
Malgré le message conciliant de ce dénouement, les censeurs gouvernementaux arrivèrent à une conclusion unanime que Les pavés sur le pavé ne pouvait être représenté. Dans un rapport long et détaillé, daté du 8 août 1850 (quelques jours à peine après le rétablissement de la censure, le 30 juillet 1850), les censeurs résumèrent ainsi la pièce : « Des regrets pour le passé, des espérances pour l'avenir promis par la divine étoile et enfin la constitution républicaine chassée à coups de balai. Voilà la pièce telle que les spectateurs l'attendront et la comprendront » (AN F/21/971). Selon les censeurs, parmi les objets balayés lors de la scène finale, figuraient la constitution républicaine, allusion ouverte aux rumeurs qui circulaient à cette époque d'un possible coup d'état par Louis-Napoléon Bonaparte.

Objet d'un deuxième rapport le 22 août et d'un troisième rapport le 29 août, la pièce put enfin être représentée avec de très nombreuses suppressions et modifications. En particulier, les auteurs durent supprimer toute référence directe aux personnalités politiques et aux rumeurs concernant un coup d'état. Un personnage attira en particulier l'attention des censeurs, celui représentant le théâtre du Vaudeville portant un costume qui rappelait celui du personnage principal de La foire aux idées (sc. 5). Ce personnage devait à l'origine chanter quelques-uns des couplets les plus connus de La foire aux idées. Les censeurs éliminèrent ces airs et obligèrent le personnage à se taire. Devenu donc muet, il finit par ne chanter que des airs anodins.

Malgré la décision de supprimer dans cette pièce les airs jugés subversifs, un compte rendu de la pièce rend clair que le public reconnaissait l'allusion à La foire aux idées : «Le Vaudeville, représenté par le personnage principal des Numéros de la Foire aux idées, arrive sans cesse sur la scène, puis, dès qu'il va parler, il entend chut ! chut ! de tous les côtés, et alors, de guerre lasse, il chante $A h$ ! vous dirai-je, maman, ou, J'ai du bon tabac, tous airs qui jusqu'à présent ont paru inoffensifs »(Le siècle, 1850.09.09). La suppression des airs en question n'a donc pas éliminé la référence intertextuelle, permettant aux auteurs par l'insertion des mots « chut ! chut ! » de faire allusion à l'acte même de censure qui avait privé leur personnage de ses paroles.

Les effrayés de Muret et Montheau, écrite en 1851, illustre encore la façon dont les censeurs cherchaient à atténuer les allusions politiques, tout en laissant aux auteurs d'ouvrages comiques une certaine latitude dans les sujets abordés. Dans cette pièce, un écrit incendiaire publié anonymement sous le titre, L'ogre couleur de sang, est répandu afin d'effrayer les habitants par de sinistres prédictions politiques. Alarmés, plusieurs propriétaires, tel Coquardeau, 
refusent des places au sein du conseil municipal parce qu'ils ont trop peur de « l'effroyable tremblement où tout va s'abîmer, s’engloutir, s'anéantir » (sc. 8) prédit par la brochure.

À cette intrigue politique se mêle le traditionnel thème du mariage à obstacles. Julien, dessinateur de la fabrique de Raimbert, veut épouser la fille de Coquardeau. Ce dernier, toujours terrifié, refuse la main de sa fille : «... songez donc si c'est la peine / de former de semblables nœuds / quand la fin du monde est prochaine !» (sc. 8). Mais le tout n'est qu'un subterfuge de la part de l'usurier Grinchard, qui cherche à jeter la terreur dans la commune dans le but de faire de bonnes affaires.

Tout se termine bien, cependant. Grinchard est démasqué et Coquardeau, rassuré, donne la main de sa fille à Julien. Malgré ce dénouement heureux, la pièce fut d'abord interdite. Tout en trouvant les sentiments exprimés par les auteurs louables, les censeurs s'inquiétèrent de la portée politique de la pièce : «depuis notre entrée en fonctions, nous avons cherché et réussi à écarter du théâtre la politique, de quelque parti qu'elle vînt » (AN, F/989, rapport du 3 novembre 1851). Malgré l'avis négatif des censeurs, la pièce fut enfin autorisée par le ministre de l'Intérieur lui-même le 27 novembre 1851. Les changements exigés ne changèrent pourtant rien au point de départ de l'intrigue. Le titre de la brochure qui sème la panique fut changé ( $L e$ dragon de feu), mais le contenu resta le même (la prédiction de calamités et d'horreurs). Les mots « le volcan bouillonne, l'explosion menace » furent remplacés par «l'univers va sauter, s'effondrer, ça se disloque, ça craque », mais l'idée d'une menace imminente restait. Les changements exigés par les censeurs ne firent qu'atténuer la portée politique de la pièce, sans changer son rapport à l'actualité du moment.

Un dernier exemple illustre la persistance des thèmes et allusions politiques, malgré les interventions des censeurs. D'abord intitulée Un mendiant qui passe, ensuite Un pauvre qui passe, la pièce Une clarinette qui passe ne fut autorisée qu'avec un titre libre de toute connotation politique. Selon les censeurs, les deux premiers titres risquaient de donner « une couleur socialiste » $(\mathrm{AN}, \mathrm{F} / 21 / 989)$ à l'œuvre qui mettait en scène le personnage du mendiant Bridoie qui porte la croix de la Légion d'honneur, gagnée en Algérie. Les censeurs jugèrent scandaleux la présence de cette médaille, la plus haute distinction accordée par l'Etat français à un de ses citoyens, sur la poitrine d'un mendiant. Dans la version finale de la pièce, Bridoie n'est plus un mendiant, mais un musicien ambulant, quelqu'un qui gagne sa vie, et il ne porte plus la croix. Le contraste entre les riches malhonnêtes de la pièce et l'honorable musicien fut ainsi 
atténué mais ne disparut pas entièrement. Comme le résuma le chroniqueur du Constitutionnel :

Une Clarinette qui passe ; c'est le titre qu'on a permis aux auteurs. À propos de ce petit vaudeville [...] il y a eu de grandes discussions dans les bureaux de la censure. MM. Labiche et Marc Michel avaient intitulé leur pièce : Un Pauvre qui passe. Au lieu de pauvre, il leur a fallu mettre clarinette; changement grave, qui fort heureusement ne touche pas au fond de l'ouvrage. (1851.01.06)

\section{Conclusion}

Que pouvons-nous conclure de ce bref tour d'horizon de quelques vaudevilles représentés pendant et après la période de liberté d'expression ? Le théâtre populaire s'était-il vraiment transformé en une «arène ouverte aux passions politiques » dès la suppression de la censure, comme le laisse entendre le rapport des censeurs ? Les censeurs réussirent-ils à faire du théâtre « un lieu de repos et de distraction » grâce à leurs interventions ?

Écrites pendant la brève période de liberté qui suivit la révolution de février 1848 , les trois premières pièces analysées dans cet article soulignent le rôle souvent méconnu du vaudeville comme miroir parodique de la société, de ses institutions et de ses personnalités marquantes. En plus de se moquer de la bourgeoisie et ses travers, ces pièces abordaient directement l'enthousiasme du public pour les banquets et clubs politiques, ainsi que les nouvelles institutions démocratiques, sans pour autant abandonner d'autres conventions du genre, tels les amants malheureux, les époux infidèles et les quiproquos. Destiné à un public mixte, le vaudeville, libéré des contraintes de la censure, rendait compte des principaux bouleversements idéologiques, artistiques et politiques qui ont rythmé la société française entre la révolution de février et 1850 .

Objet d'une attention accrue après le retour de la censure, le vaudeville continuait à offrir, à travers son prisme parodique, un reflet des principaux changements sociaux et politiques survenus en France pendant les premières années du Second Empire. Atténuées, ces allusions ne perdirent que peu de leur mordant et de leur effet galvanisant sur le public. Les efforts considérables déployés par les censeurs pour les atténuer nous en disent long sur l'incertitude d'un nouveau régime qui cherchait encore à consolider son pouvoir dans les rues de Paris, comme sur ses principales scènes. Trop attachée à la correction des manuscrits et de la parole écrite, la censure se révéla impuissante face à la polyvalence du vaudeville, sa musique, ses airs empruntés et son intertextualité. Pour finir, les transformations du baron Haussmann, qui poussèrent la population ouvrière vers les banlieues de la capitale, eurent plus d'impact, rendant 
le public théâtral plus homogène et les représentations moins houleuses.

La création, en 1851, de la Société des auteurs, compositeurs et éditeurs de la musique finit par achever cette transformation. Avant la création de cette société, les auteurs et les compositeurs de musique n'étaient pas rétribués lorsque leurs créations étaient interprétées en public, ce qui donnait aux vaudevillistes la possibilité de reprendre comme bon il leur semblait la musique d'autrui (Lemarchand 13-15). À partir de 1851, cependant, le nombre d'airs que les vaudevillistes pouvaient emprunter fut de plus en plus limité. Pendant cette période de transition, on voit petit à petit disparaître les «timbres » traditionnels au profit d'airs nouvellement composés pour la pièce. Le vaudeville évolua dès lors vers la comédie de situation et de mœurs, sans perdre pour autant son attachement à la parodie des tendances du jour et des actualités.

\section{Bibliographie}

Bara, Olivier. «La folie du vaudeville face à la raison de la censure sous la Monarchie de Juillet ». Teatro do Mundo. Teatro e Censura. Faculdade de Letras. Universidade do Porto, 2013. 139.

Best, Janice. «"Le privilège de la parodie" : la censure politique dans quatre vaudevilles de Bayard, Kock, Labiche, et Royer ». Nineteenth-Century French Studies 44.3-4 (2016). 250-265.

Krakovitch, Odile. La censure théâtrale (1835-1849). Édition des procès-verbaux. Paris: Classiques Garnier, 2016.

Hemmings, F.W.J. Théâtre State in France 1760-1905. Cambridge : Cambridge UP, 1994.

Lemarchand, Henry. «Merci, Ernest Bourget ». La Sacem aujourd'hui. Paris : Sacem, 1977. 1315.

Malandain, Gilles. «Quel théâtre pour la République ? Victor Hugo et ses pairs devant le Conseil d'état en 1849 ». Sociétés et représentations 11.1 (2001). 206.

Martin, Roxane. «Mélodrames et vaudevilles ». Le théâtre français du XIX $X^{e}$ siècle. Dir. Hélène Laplace-Claverie, Sylvain Ledda et Florence Naugrette. Paris : Avant-scène théâtre, 2008. 68-93.

Michaud, dir. «Éléonore Tenaille de Vaulabelle ». Biographie universelle ancienne et moderne. t. 95. Paris : Beck, 1862. 209-211.

Terni, Jennifer. «A Genre for Early Mass Culture : French Vaudeville and the City, 18301848 ». Theatre Journal 58.2 (2006). 221-48. 
Yon, Jean-Claude. Les spectacles sous le Second Empire. Paris : Colin, 2010.

\section{NOTES}

${ }^{1}$ Odile Krakovitch affirme que cette pièce a été interdite «sans laisser de traces », mais la pièce a bien été jouée, comme en atteste le compte rendu paru dans Le siècle du 9 septembre 1850.

${ }^{2}$ Proudhon portait, en effet, des lunettes.

${ }^{3}$ En réalité, un des pseudonymes de Jules Cordier, de son vrai nom Éléonore Tenaille de Vaulabelle. Vaulabelle fut un écrivain et auteur dramatique français (1801-1859) qui publia des romans sous le pseudonyme d'Ernest Desprez et des pièces de théâtre principalement sous le pseudonyme de Jules Cordier.

${ }^{4}$ La loi sur la presse du 9 septembre 1835 mit fin à la législation libérale adoptée au lendemain de la révolution de 1830. Cette loi fut particulièrement sévère pour les journaux. Le nombre de condamnations pour offense à la personne du roi, pour incitation à la révolte, pour outrage et injures envers des fonctionnaires détenteurs de l'autorité publique augmenta considérablement après 1835 . 